\title{
In silico and saturation transfer difference NMR approaches to unravel the binding mode of an andrographolide derivative to K-Ras oncoprotein
}

\begin{abstract}
Background: Andrographolide and its benzylidene derivatives, SRJ09 and SRJ23, potentially bind oncogenic K-Ras to exert anticancer activity. Their molecular interactions with K-Ras oncoproteins that lead to effective biological activity are of major interest. Methods \& results: In silico docking and molecular dynamics simulation were performed using Glide and Desmond, respectively; while saturation transfer difference NMR was performed using GDPbound K-RasG12V. SRJ23 was found to bind strongly and selectively to K-RasG12V, by anchoring to a binding pocket (namely p2) principally via hydrogen bond and hydrophobic interactions. The saturation transfer difference NMR analysis revealed the proximity of protons of functional moieties in SRJ23 to K-RasG12V, suggesting positive binding. Conclusion: SRJ23 binds strongly and interacts stably with K-RasG12V to exhibit its inhibitory activity.
\end{abstract}

Keyword: Desmond; Glide; K-Ras oncoprotein; SRJ23; STD-NMR; Andrographolide; Anticancer; Binding pocket; Docking; Molecular dynamics 